$27^{e}$ Cours international de Justice constitutionnelle : Constitution et sécurité extérieure Mercredi 2 et jeudi 3 septembre 2015

\title{
Les pouvoirs du Chef de l'État dans le recours aux opérations extérieures
}

\author{
par \\ Christian BEHRENDT \\ Professeur à l'Université de Liège et au Collège de Défense de l'École Royale Militaire de Belgique \\ Assesseur au Conseil d'État de Belgique
}

\section{Introduction}

«Les pouvoirs du Chef de l'État dans le recours aux opérations extérieures »: si nous laissons vagabonder un instant notre esprit à la lecture de ce titre, nous pourrions songer, au détour d'une pensée, à un tout autre thème, en apparence sans rapport direct et immédiat, à savoir à l'art.

Le monde artistique, que ce soit à travers les peintures, les chansons épiques et les sculptures, a en effet illustré l'action guerrière et nous a d'ailleurs bien souvent habitués à représenter, en première ligne d'une bataille, le Prince vaillant qui harangue ses foules et mène l'assaut décisif en brandissant son épée. L'Histoire regorge de ces moments immortalisés par des artistes où le Monarque ou l'Empereur se tient physiquement aux 
côtés de ses armées et conduit personnellement les assauts sur le terrain ${ }^{1}$. Autant l'avouer d'emblée, il serait malaisé d'imaginer, de nos jours, les dirigeants sur de majestueux chevaux blancs en train de donner dans les lueurs du petit matin les ultimes ordres du haut d'une colline. Pourtant, même si la nature des conflits a évolué, le rôle du Chef de l'État demeure bel et bien important - pour ne pas dire central - en cette matière.

Notre présentation sera divisée en deux parties, deux angles d'attaque, sans mauvais jeu de mots. La première sera dédiée aux prérogatives du Chef de l'État, qui représente le Pouvoir exécutif, face aux deux autres Pouvoirs, autrement dit face aux impératifs démocratiques des systèmes occidentaux actuels et face aux différents mécanismes de mise en œuvre d'une éventuelle responsabilité, notamment juridictionnelle. La dernière partie se concentrera sur les prérogatives du Chef de l'État au sein même du Pouvoir exécutif, plus spécifiquement dans ses rapports avec l'armée proprement dite qui doit, en tout cas dans la conception démocratique occidentale, lui être institutionnellement et organiquement soumise. Si la première partie concerne plus exactement les interactions horizontales, avec les deux autres Pouvoirs, la seconde est donc pensée dans un sens plus vertical.

\section{Remarques liminaires}

A titre liminaire, il convient de lever le voile de toute équivoque et d'analyser la portée des termes qui constituent le cœur de nos réflexions, les pouvoirs du Chef de l'État dans le recours aux opérations extérieures, étant entendu que méconnaître certaines données élémentaires risquerait de compromettre notre méthodologie d'étude et de constituer une pierre d'achoppement dans notre raisonnement.

Selon le dictionnaire juridique CoRnu, l'expression Chef de l'État se définit comme :

« l'autorité, l'individu ou (plus rarement) le collège dont l'intervention dans la procédure d'élaboration des actes juridiques les plus importants relevant surtout du pouvoir exécutif (par exemple, la promulgation des lois, la ratification des traités) ou la présence à certaines cérémonies marquent de manière symbolique que c'est à l'État qu'il convient d'imputer ces conduites [...]

\footnotetext{
1 « Il va sans dire, la représentation de la guerre a certainement évolué au fil des siècles, non seulement parce que les supports préférés ont changé, par exemple, des décorations architecturales aux mosaïques murales et aux manuscrits enluminés, mais aussi parce que la compréhension de la guerre a changé au fil des siècles. Une des rares constantes, toutefois, était et demeure la "valeur de propagande" de la représentation de la guerre. Qu'il s'agisse des peintures murales, telles que la représentation de la victoire de Ramsès III à la bataille de Qadesh, les scènes de bataille sculptées sur la Colonne de Trajan ou de la peinture à l'huile de Napoléon à la bataille des pyramides, leur but reste le même : une glorification d'un chef militaire ou une célébration des exploits militaires » (voy. S. TzU et V. ChARLES, L'art de la guerre, New York, Parkstone International, 2012, p.
} 8). 
elle peut, selon les régimes, disposer de pouvoirs politiques réels et importants, comme par exemple le Président des États-Unis ou, en France, le Président de la République sous la Ve République ou réduits, comme au Royaume-Uni ».

Ce rappel n'est pas inutile puisque l'énoncé au cœur de la discussion n'est pas «les pouvoirs du Chef de gouvernement dans le recours aux opérations extérieures », ni "les pouvoirs du Gouvernement, dans son ensemble», pas plus, à première vue, que "les pouvoirs du Pouvoir exécutif». Ici, selon le libellé, c'est le Chef de l'État. Or, le pouvoir que peut détenir le Chef d'État varie foncièrement d'un régime à l'autre. Si nous opérons un détour sommaire par la distinction à opérer entre les trois formes de gouvernement qui existent, les régimes semi-présidentiel, présidentiel et parlementaire, nous réalisons qu'une importante difficulté surgit puisque chacun de ces régimes octroie par principe des rôles et missions fondamentalement différents au Chef de l'État ${ }^{2}$.

Un régime parlementaire, tel que le Royaume-Uni - patrie par excellence du parlementarisme -, la République fédérale d'Allemagne ou encore le Royaume de Belgique, consacre la révocabilité mutuelle des deux pouvoirs, exécutif et législatif. Le pouvoir législatif a le pouvoir de renverser le gouvernement, dirigé par un chef du gouvernement. Le Chef de l'État, élu par les chambres ou désigné par la règle dynastique dans les monarchies, a en général le droit de dissoudre la ou les assemblées qui composent le Pouvoir législatif. Le chef du gouvernement est nommé par le Chef de l'État, mais est - ceci est la marque de fabrique du régime - politiquement responsable devant le Parlement. Un régime présidentiel, comme les États-Unis, se caractérise par une nette séparation entre les pouvoirs exécutif et législatif. Le Président - Chef de l'État et de gouvernement - nomme de façon discrétionnaire les membres qui constituent son gouvernement, sans égards pour le Parlement qui ne dispose d'aucune prérogative de motion de censure à leur égard. Un régime semi-présidentiel, par exemple la République polonaise ou la Ve République française, peut être décrit comme un système hybride, à mi-chemin entre les deux modèles précédemment décrits. La double révocabilité des pouvoirs est conservée tandis qu'une dualité de l'exécutif est assurée par la présence d'un chef de gouvernement et d'un Chef de l'État. Cependant, le président est généralement élu directement par suffrage universel et est pourvu de pouvoirs personnels.

On l'aura compris, sur le plan militaire, le rôle que joue le Président de la Ve République diverge fondamentalement de celui assuré par la Reine du Royaume-Uni de GrandeBretagne et d'Irlande du Nord ou par le Président de la République fédérale d'Allemagne. Nous sommes donc confrontés à la difficulté qu'interpréter strictement l'expression «Chef de l'État » nous amènerait à ne prendre en compte que les régimes présidentiels et semiprésidentiels où le Chef de l'État jouit réellement de pouvoirs effectifs puisque, dans les régimes dits parlementaires, la personne qui importe de facto, c'est le chef de gouvernement. Au Royaume-Uni, que ce soit lors de la seconde guerre mondiale ou lors de

2 Voy, par exemple C. BeHREndT et F. BOUHON, Introduction à la Théorie générale de l'État, 3e éd., Bruxelles, Larcier, 2014, pp. 280-298. 
la guerre des Malouines (Falklands War en anglais, Guerra de las Malvinas en espagnol), les Premiers ministres de l'époque, respectivement Winston CHURCHILL ${ }^{3}$ et Margaret THATCHER ${ }^{4}$, étaient aux commandes des opérations. A titre d'exemple, à nouveau, selon l'article 37 de la Constitution du Royaume de Belgique: «[a]u Roi appartient le pouvoir exécutif fédéral, tel qu'il est réglé par la Constitution ». Le libellé de la disposition semble limpide, alors que c'est bien le gouvernement fédéral, avec à sa tête le Premier ministre, qui dirige l'action du gouvernement. Le Chef de l'État, le Roi - personne physique - joue il est vrai un rôle par la sanction royale, c'est-à-dire l'acte par lequel le Roi marque son accord formel avec la loi, et la promulgation, acte par lequel le Roi confirme l'existence de la loi, mais ce rôle est limité, sans commune mesure avec les pouvoirs du chef de gouvernement.

Une lecture stricte de notre thème nous priverait de la sorte d'un nombre imposant d'États dans notre aperçu comparatif. Aussi, dans l'optique de mettre ces trois structures gouvernementales en comparaison, tout au long de notre propos, il conviendra d'avoir l'acception la plus large, et ainsi la plus utile, du concept de «Chef de l'État». En effet, pour être équitable en droit comparé, et ne pas se priver d'emblée de l'examen de nombreux États de la planète - à savoir les pays à structure parlementaire -, il nous paraît nécessaire d'assimiler le «Chef de l'État» au Pouvoir exécutif dans sa globalité, pour nous permettre d'intégrer tous les États dans le schéma comparatif5.

Cela étant précisé, nous devons revenir à présent sur l'expression «opération extérieure ». Les Opex sont, dans le vocabulaire du Ministère français de la Défense nationale, des interventions des forces militaires françaises - ou, disons-le de manière plus conceptuelle, nationales - en dehors du territoire national qui peuvent se dérouler en collaboration avec les organisations internationales, telles que l'Organisation des Nations Unies et l'Organisation du Traité de l'Atlantique nord, et parfois avec des armées locales. Il s'agit plus généralement de l'emploi des forces armées mobilisées dans un contexte où il existe une menace ou un risque pour l'intégrité physique des troupes armées engagées dans l'opération extérieure. Il est important de souligner que l'expression «opérations extérieures » recouvre ainsi une multitude de réalités, parfaitement hétéroclites ${ }^{6}$.

En Europe, la guerre froide a surtout été caractérisée par le recrutement et le déploiement de troupes conventionnelles, professionnelles et massives, entrainées à défendre l'intégrité et la souveraineté du territoire face à un ennemi, qui présentait - nonobstant les différences idéologiques - les mêmes traits organisationnels, en cas de conflit total et, également, conventionnel qui impliquerait la mobilisation de toutes les ressources des

${ }^{3}$ I. JEnnings, The British Constitution, 5e éd., Cambridge, University Press, 1971, p. 188.

${ }^{4}$ R. Rose, Politics in England: Change and Perspective, 5e éd., Basingstoke, Macmillan, 1989, p. 97.

5 Voy. d'ailleurs, en ce qui concerne l'expression "the crown», P. LEYLAND, The Constitution of the United Kingdom, A contextual Analysis, 2e. éd., Oxford, Hart Publishing, 2012, p. 98.

${ }^{6}$ G.-M. Chauveau et H. Gaymard, Rapport d'information déposé en application de l'article 145 du règlement, par la commission des affaires étrangères, en conclusion des travaux d'une mission d'information constituée le 11 décembre 2013 sur la mission "Engagement et diplomatie" n²777 déposé le 20 mai 2015 (mis en ligne le 4 juin 2015), Documents d'information de l'Assemblée nationale, Paris, Assemblée nationale, 2015, p. 16. 
belligérants dès le déclenchement des hostilités. "L'ensemble du champ conflictuel était ainsi référé à ce paradigme ", écrivent Etienne de DURAND, Marc HECKER et Aline LEBOEuf, « et les conflits virtuels ou actuels, [étaient] décrits comme de «haute», «moyenne » ou "basse» intensité, selon leur proximité avec l'affrontement central, les risques qu'ils entraînaient à l'échelle globale et donc leur position sur l'échelle d'escalade ${ }^{7}$. Or, la grande - et destructrice - confrontation entre les deux blocs n'a pas eu lieu. Elle semble aujourd'hui appartenir au passé. Pourtant, cela n'a pas empêché la survenance de conflits locaux, entre pays, mais aussi internes à ceux-ci, qui se sont multipliés en présentant une nouvelle forme de complexité ${ }^{8}$. Face à ces crises géo-localisées, l'enjeu est, depuis une trentaine d'années, non de vaincre militairement un adversaire, mais de résoudre des conflits violents dont les populations civiles sont les premières victimes, que ce soit lors d'interventions dans le cadre d'accords de défense, d'opérations de rétablissement et de maintien de la paix, des missions de lutte contre le terrorisme, de sauvetage des ressortissants en danger, etc. L'adversaire à neutraliser a en quelque sorte changé fondamentalement de nature. Il est devenu un acteur séparé d'un territoire déterminé, des frontières nationales classiques, et il présente des motivations, volontés, religions et idéologies diverses et variées ${ }^{9}$. La doctrine des armées françaises, comme celle des armées occidentales, a dû assimiler ce postulat et distinguer "maîtrise de la violence » et "coercition de force». Classiquement, tandis que la puissance aérienne et les forces spéciales assurent à l'aide des outils technologiques et stratégiques, le bon déroulement de la phase offensive, les forces terrestres se déploient sur le terrain pour garantir la pacification des échanges ${ }^{10}$.

Plusieurs questions, inhérentes à tout régime démocratique, qui postule la prééminence du pouvoir civil sur les militaires ${ }^{11}$, s'imposent. Deux grandes thématiques qui transcendent l'organisation structurelle de la Défense nationale seront ainsi traitées : premièrement, le Chef de l'État face aux contraintes externes au pouvoir exécutif, et deuxièmement, le Chef de l'État face à la structure interne, soit face au pouvoir militaire.

\footnotetext{
7 E. DE Durand, M. HeCKer et A. Leboeuf, «Des Balkans à l'Afghanistan : les opérations de stabilisation complexes », Politique étrangère, 2005, p. 330.

8 Voy. à ce sujet M. VAÏSSE, Les relations internationales depuis 1945, 13e éd, Paris, Dunod, 2013, pp. 283-292.

9 T. RiD et M. HECKER, War 2.0: Irregular Warfare in the Information Age, Westport-London, Praeger security International, 2009, p. 280.

${ }^{10}$ E. DE DurAnd, M. HeCKER et A. Leboeuf, op. cit., p. 330 ; Voy. aussi D. RESTEIGNE, « Des militaires devenus sans frontières? ?, Pyramides, 2011, pp. 77-93.

11 S. COHEN, « Le pouvoir politique et l'armée », Pouvoirs, 2/2008, p. 19.
} 


\section{1 ère Partie : Le Chef de l'État face aux autres Pouvoirs}

\section{Cedant arma togae. L'armée cède à la toge $\mathrm{e}^{12}$.}

Cette maxime, qui provient du premier hémistiche d'un vers de Cicéron pour son propre hommage, synthétise la tradition démocratique occidentale ${ }^{13}$ qui postule le principe de la primauté du pouvoir civil et politique sur l'institution militaire ou, pour reprendre l'expression d'Aristide BRIAND, de signaler qu'est placée entre les mains du Chef de l'État « la conduite politique de la guerre et le contrôle des opérations militaires ${ }^{14}$. En outre, l'adage romain rappelle aussi que le Chef de l'État est, dans un même temps, chef des armées. Il est pratiquement universel que le pouvoir exécutif exerce la charge d'assurer la défense de la population et de la Cité. Michel LIEGEOIS remarque à ce titre « qu'au-delà de la variété des régimes constitutionnels, la décision d'emploi de la force armée est un domaine de l'action politique dans lequel le plus haut niveau de l'État jouit d'un monopole » 15 .

Vers l'an mille avant Jésus Christ, les peuplades israélites disent au prophète Samuel, pour combattre les Philistins : "Nous aurons un roi pour conduire nos guerres ». Dans la Grèce et la Rome antiques, en plus du pouvoir civil, le chef de l'exécutif possède la force militaire de manière totale sans qu'il lui soit permis d'utiliser l'armée à l'intérieur de l'espace métropolitain où il ne dispose que du pouvoir civil. Dans cette configuration, l'armée ne s'occupe que de l'ennemi extérieur ${ }^{16}$. Durant le Moyen-Âge, les sacres des rois de France dans la cathédrale de Reims ont notamment pour moment symbolique la transmission au nouveau souverain de l' "épée de Charlemagne », également dénommée Joyeuse, comme symbole de la détention d'un pouvoir militaire ${ }^{17}$. L'émergence des États-Nations à la suite du Traité de Westphalie n'a pas changé les choses.

Chargé de préserver l'intégrité du territoire, le souverain a comme première mission la défense et le contrôle des troupes. Pour HAURIOU, " [l]e pouvoir des fondateurs de l'État est à la fois civil et militaire; très probablement, le régime d'État a pris naissance dans les villes refuges où les premières populations agricoles étaient obligées de s'abriter contre les incursions des tribus pillardes. Dans ces espèces de camps de concentration, il y avait une sorte d'état de siège, c'est-à-dire que des populations civiles étaient momentanément

\footnotetext{
12 CiCERon, De officiis (Des devoirs), I, 22.

${ }^{13}$ F. Fisson-Roche, « Article 15 », in : F. Luchaire, G. ConAC et X. Pretot (sous la dir. de), La Constitution de la République française - Analyses et commentaires, 3e éd., Paris, Economica, 2009, p. 504.

14 Annales de la Chambre des députés, 1916, pp. 134-135.

15 M. LIEGEOIS, « L'instrument militaire dans la politique étrangère » in : C. RoosEns, V. Rosoux et T. DE WILDE D’EsTMAEL, La politique étrangère, le modèle classique à l'épreuve, Bruxelles, P.I.E.-Peter Lang, 2004, p. 406.

${ }^{16}$ Cette technique de cantonnement de l'armée a été violée à Rome par Jules César en franchissant le Rubicon. ${ }^{17}$ L'épée utilisée lors du sacre des rois de France portait bien le même nom que l'épée de Charlemagne, mais tout indique qu'elle a été constituée à partir d'éléments d'époques différentes.
} 
soumises à l'autorité d'un chef militaire. La période de guerres continuelles qui accompagna la formation des nations et leur fixation au sol conserva à l'État son caractère militaire » ${ }^{18}$. Sur la base de la pensée du grand juriste français, Jean-Christophe VIDELIN en conclura que « [1]a détention du pouvoir militaire ne suffit pas. Elle doit être en plus exclusive » 19 et que " [c]'est pour cette raison que dans tous les États modernes, le chef de l'État est le chef des armées. Il symbolise en sa personne la centralisation du pouvoir militaire $»^{20}$. L'État s'est donc véritablement constitué à travers son chef militaire devenu chef politique, soumettant l'armée à sa volonté politique et détenteur du monopole de la force légitime. Nous pouvons faire référence à Emile DE GIRARDIN qui écrivait, en 1867, « [s]i la guerre n'avait jamais existé, il n'existerait pas de nations : les nations sont filles de la guerre. En effet, une nation n'est qu'un faisceau d'habitants unis par la nécessité de se défendre contre le danger d'être conquis et enlevés à ce qu'ils considèrent, à tort ou à raison, comme leur indépendance $»^{21}$.

Dans les démocraties modernes, il va sans dire que le gouvernement civil jouit de l'autorité sur l'armée. Cette soumission de l'armée permet de la cantonner - physiquement ou organiquement - afin qu'elle ne constitue pas une menace mais un outil pour l'État. Pour s'en assurer, ce principe est constitutionnellement organisé tant en désignant le Chef de l'État, chef des armées, qu'en prévoyant les fonctions précises et encadrées de celles-ci. Le rôle du Chef de l'État dans les opérations militaires dépend des exigences démocratiques, inséparables du fonctionnement de l'État contemporain et pacifique. Selon T. ClifTON MORGAN et Sally HOWARD CAMPBELL, « leaders who have to stand for popular election should be expected to take public attitudes into account when making decisions ${ }^{22}$. D'ailleurs, comme le soulignent Bruce RUSSETT et John ONEAL : « leaders of democratic states typically experience high political costs from fighting wars - always from losing them, and often despite winning them $»^{23}$.

${ }^{18}$ M. Hauriou, Principes de droit public, 2e éd., Paris, Recueil Sirey, 1916, pp. 443-444.

19 J. C. Videlin, Droit de la défense nationale, $2^{\mathrm{e}}$ éd., Bruxelles, Bruylant, 2014, p. 38.

20 Ibid., p. 38.

${ }^{21}$ E. DE GIRARDin, La politique universelle, Décrets de l'avenir, 2e éd., Paris, Librairie nouvelle, 1854, p. 29 ; voy également J.-Y. DoRMAGEN et D. MouchAR, Introduction à la sociologie politique, Bruxelles, De Boeck, 2008 p. 34 : «il découle de ce processus historique que le grand moteur de la construction étatique est la guerre, qu'il s'agisse des guerres entre seigneurs rivaux, dont on vient de voir qu'elles conduisaient à l'émergence d'un pouvoir central, ou des guerres avec des puissances étrangères qui jouent, elles aussi, un rôle essentiel dans la consolidation et l'institutionnalisation de l'État».

22 T. C. Morgan et S. Howard Campbell, « Domestic Structure, Decisional Constraints, and War - So Why Kant Democracies Fight? », Journal of Conflict Resolution, 1991, p. 190.

23 B. RussetT et J. OnEal, Peace : Democracy, Interdependence, and International Organizations, New York, Norton, 2001, p. 54. 


\section{A. Les relations entre le Pouvoir exécutif et le Pouvoir législatif}

Cela étant dit, parmi les États occidentaux, les interactions entre les pouvoirs exécutif et législatif varient intensément.

Dans les régimes parlementaires, les ministres ont hérité des prérogatives suprêmes en matières militaires. Au fur et à mesure des décennies, nous avons été témoin d'un fort recul de la "prérogative royale», c'est-à-dire de la capacité d'initiative politique concrète du monarque, avec l'affirmation exponentielle des pouvoirs du Premier ministre et de la responsabilité gouvernementale devant le Parlement ${ }^{24}$. Ce n'est désormais plus qu'en cas de crise, lorsque les clivages partisans sont affaiblis, que le souverain - ou le président peut, en tant que médiateur entre les formations politiques, éventuellement parvenir à jouer un rôle de décideur.

$\mathrm{Au}$ Royaume-Uni, le Roi ne pouvant rien faire sans contreseing (ministerial countersignature en anglais), il ne peut pas non plus mal faire (the king can do no wrong), bien que ce soit à lui que revienne la fonction de chef des armées ${ }^{25}$. Si le titre de commandant en chef est rarement utilisé par le Souverain, il est correct de le décrire de la sorte en tant que figure tutélaire doté prérogatives royales parmi lesquelles figures l'envoi de troupes armées ${ }^{26}$. Pourtant, vu la longue tradition parlementaire du pays, c'est le Premier ministre du Royaume-Uni qui détient de facto le commandement des Forces armées britanniques à l'aide du cabinet, noyau dur du pouvoir exécutif. Le ministère de la Défense (Ministry of Defence) est, quant à lui, le département gouvernemental chargé de prévoir et d'exécuter la politique de défense de la nation à travers les forces armées. Ce ministère est dirigé par le secrétaire d'État à la Défense (Secretary of State for Defence) et par trois responsables parlementaires : le ministre d'État aux forces armées (Minister of State for the Armed Forces), le ministre pour l'approvisionnement militaire (Minister for Defence Procurement) et le ministre des vétérans (Minister for Veterans' Affairs). Il faut ajouter à cette structure plusieurs comités et conseils chargés de la gestion concrète des armées, comme le Defence Council (Conseil de la défense), le Chiefs of Staff Committee (Comité des Chefs d'État-Major), le Defence Management Board (Conseil d'administration de la défense), ainsi que trois conseils uni-arme (Army Board pour la British Army, Admiralty Board pour la Royal Navy et Air Force Board pour la Royal Air Force) sous la direction du secrétaire d'État à la Défense. Le premier ministre assure seul la liaison entre

\footnotetext{
${ }^{24}$ P. ChASSAIGNE, « La société britannique, la monarchie et la guerre, 1914-1945 », Histoire, économie et société, 2004, no 2 : La société, la guerre, la paix, 1911-1946, pp. 182-183.

${ }^{25}$ P. LEYLAND, op. cit., p. 97.

26 C. Turpin et A. Tomkins, British Government and the Constitution: Text and Materials, 6e éd., Cambridge, Cambridge University Press, 2007, p. 163.
} 
le gouvernement et le monarque. C'est lui qui se rend chaque semaine auprès du roi qui, depuis le règne de GEORGE Ier, n'assiste plus aux délibérations du cabinet ${ }^{27}$.

Clef de voûte du système institutionnel britannique, même si le « monarque règne mais ne gouverne pas », la monarchie anglaise ne jouit pas d'une mission extraordinaire dans le cadre des conflits armés. La monarchie n'a d'ailleurs pas pu échapper à la "mise à l'épreuve " que constituèrent les deux guerres mondiales ${ }^{28}$ au cours desquelles les interventions du souverain sont rares, et généralement symboliques. Au cours de la Première guerre mondiale, GEORGE V n'est intervenu directement qu'en trois occasions : en 1915, en organisant la conférence de Buckingham Palace sur la question de la conscription ; en décembre 1916, en refusant la dissolution que le leader conservateur Bonar LAW a mis comme préalable à son acceptation du poste de Premier ministre, enfin en soutenant publiquement le général HAIG, commandant en chef des troupes britanniques, dont les choix tactiques et stratégiques ont fait l'objet de controverses. Aussi, le roi GEORGE V n'a donc pas assuré de façon effective le commandement des opérations, bien que l'enrôlement des volontaires se soit fait sous son égide ("Your king and your country need you » qui évolua ensuite en "For King and Country »). La fonction de père tutélaire de ses sujets a beaucoup plus été développée. A titre d'exemple, en 1917, le Roi a décidé de changer le nom de la dynastie de "Saxe-Cobourg» en "Windsor», pour faire oublier les origines en partie germaniques de la maison royale et ses liens avec nombre de dynasties allemandes. $\mathrm{Au}$ cours de la Seconde guerre mondiale, les choses ont été encore moins perceptibles.

Autrement dit, c'est au Premier ministre que revient la charge de diriger les opérations, tout en étant responsable devant son parti et les électeurs. Cela étant dit, le monarque préserve son droit, en tant que fountain of honours, de décerner les honneurs. Enfin, en théorie pure, la monarchie conserve également le pouvoir de prévenir l'utilisation inconstitutionnelle de l'option militaire.

La Chambre des Communes n'a dans la rigueur des principes aucune prérogative pour autoriser ou contrer le déploiement de troupes à l'étranger ${ }^{29}$. Cela étant, le 18 mars 2003, un vote de la Chambre des Communes a apporté une résolution selon laquelle elle :

« supports the decision of Her Majesty's Government that the United Kingdom should use all means necessary to ensure the disarmament of Iraq's weapons of mass destruction $»^{30}$.

\footnotetext{
${ }^{27}$ A. BERTRAND, Les techniques du travail gouvernemental dans l'état moderne: Étude de sciences politique et administrative et de droit constitutionnel comparé, Bruxelles, Institut international des sciences administratives, 1954 , p. 28,.

28 P. ChASSAIGNE, op. cit., p. 182.

${ }^{29}$ House of Lords Select Committee on the Constitution, Waging war (fifteenth Report, Session 2005-06, HL 236, London, HMSO, 206, p. 39, voy. L. Webley et H. SAmuels, Complete Public law : Text, Cases, and Materials, 3e éd., Oxford, Oxford University Press, 2015, p. 179.

${ }^{30}$ House of Commons, Deb Vol 401, col 760, 18 mars 2003.
} 
Ce vote aurait pu ne pas avoir lieu car il reste purement facultatif et à la discrétion du Premier ministre qui aurait pu passer outre en cas de vote négatif 31 , bien que le coût politique aurait sans conteste été extrêmement élevé. Par ailleurs, en août 2013, David CAMERON a bel et bien suivi la décision prise par la Chambre des communes britannique de s'opposer à une participation du pays à une action militaire en Syrie ${ }^{32}$, ce qui représente un cas inédit depuis 240 ans de rejet par le parlement d'une proposition du gouvernement de lancer une intervention militaire. En effet, c'est en 1782 que la Chambre des Communes s'est prononcée contre une poursuite des hostilités en Amérique après la capitulation de Lord CORNWALlIS à Yorktown, le 19 octobre 1781. Le roi GEORGE III, ainsi que son premier ministre, Lord NoRTH, n'ont pu convaincre le législateur britannique de continuer la guerre contre les insurgés américains. Néanmoins, en règle générale, la décision de déployer des forces à l'étranger est prise par le Prime minister, quelques conseillers et les membres les plus fiables du gouvernement, et simplement annoncé au Parlement. Par contre, chose notable, depuis la Bill of Rights de 1689, il est interdit de maintenir une armée en tant de paix sans l'aval du parlement ${ }^{33}$ à travers une loi, annuelle, dénommée le Mutiny Act.

La chose est similaire en Belgique où la Constitution précise clairement, en son article 167 $\S 1$, al. 2, que le Roi

« commande les forces armées, et constate l'état de guerre ainsi que la fin des hostilités. Il en donne connaissance aux Chambres aussitôt que l'intérêt et la sûreté de l'État le permettent, en y joignant les communications convenables ».

Il jouit à ce titre du commandement éminent, c'est-à-dire du pouvoir d'engager des troupes et de les retirer ${ }^{34}$. Cependant, une nouvelle fois, c'est bien le gouvernement qui mène la politique extérieure de défense. Le pouvoir du dernier mot lui revient, même si sa décision peut provenir, ou non, de l'opinion royale. Les acteurs principaux du processus décisionnel sont, au sein du gouvernement fédéral, le ministre des Affaires étrangères et le ministre de la Défense ${ }^{35}$. Ainsi, le fait que le Parlement n'ait joué dans la prise de décision d'engager l'armée belge au Kosovo ou contre l'État islamique qu'un rôle limité est conforme au régime de commandement issu de la Constitution ${ }^{36}$. Il ne peut intervenir qu'a posteriori, grâce au contrôle politique de l'action du gouvernement ${ }^{37}$. Cela étant, le Parlement est en

31 Cela étant, il a été soutenu qu'une coutume s'installait à l'égard de l'usage de la force à l'issue de ce vote, selon laquelle la démocratie moderne exigeait l'obtention de l'accord du parlement (voy. Lord WILSON OF Dinton, « The Robustness of Conventions in a Time of Modernisation and Change », Public Law, 2004, p. 414).

32 L. WEBlEY et H. SAMUELS, op. cit., 2015, p. 167.

${ }^{33}$ H. BARnETT, Constitutional and Administrative Law, 7e éd., New York, Routledge-Cavendish, 2009, p. 119.

${ }^{34}$ Voy. P. D'ARGENT, « Le Roi commande les forces armées », Revue belge de droit international, 1994, p. 212 et s. ; voy. également Y. LEjEune, Droit constitutionnel belge, 2e. éd., Bruxelles, Larcier, 2014, p. 550.

35 V. MOYSE et A. DumOulin, «Le processus décisionnel belge en matière d'opération civilo-militaires », Courrier hebdomadaire du CRISP, 2011/1-2, n²086-2087, pp. 5-64.

36 F. DuBuisson, «La participation de l'armée belge à l'opération «force Alliée » de l'OTAN au Kosovo : Questions au regard de l'article 167 de la Constitution », A.P.T., 2001, p. 51 et s.

37 R. ERGEC, Introduction au droit public, 2e éd., Story-Scientia, 1994, t. 1, p. 187 et R. ERGEC, «La crise du Golfe et le droit public des opérations militaires», J.T., 1991, p. 138. 
principe informé de la décision de l'exécutif de mettre en œuvre l'armée belge par le biais d'une communication gouvernementale, généralement suivie d'un vote de confiance ${ }^{38}$. L'obligation de porter à la connaissance de la Chambre et du Sénat les actes de nature politique «aussitôt que l'intérêt et la sûreté de l'État le permettent » ${ }^{39}$ ne couvre pas les décisions de gestion quotidienne ${ }^{40}$.

En Allemagne, autre régime parlementaire, la procédure se veut rigoureuse, la guerre étant explicitement interdite depuis la fin de la Seconde Guerre Mondiale ${ }^{41}$. Certes, en 1955, il y eut la formation d'une armée en Allemagne de l'Ouest, la Bundeswehr, et, en 1956, d'une force armée en Allemagne de l'Est, la Nationale Volksarmee ${ }^{42}$, mais les deux armées constituées en pleine guerre froide se limitaient, avant la chute du mur, à des entraînements et des Katastropheneinsatz. Les choses évoluèrent à la suite de la réunification ${ }^{43}$.

Evénement notable, dans son arrêt du 12 juillet $1994^{44}$, alors que l’Europe était confrontée à la crise de Yougoslavie, la Cour constitutionnelle a déclaré les opérations extérieures conformes à la Constitution du moment qu'elles sont menées dans le cadre de son article $87 \mathrm{a}$, soit la défense du territoire, ou dans le cadre d'une participation à un système de défense mutuelle collective selon l'article 24, l'état de défense englobant également des

\footnotetext{
38 A ce sujet, voy. E. VAndenbossche, "De federale Kamers en de buitenlandse missies van militairen », Chroniques de droit public, 2011, pp. 424-442

${ }^{39}$ Voy. également Y. LEJEUNE, op. cit., p. 550.

${ }^{40}$ Rapport CEREXHE, Doc. parl., Sénat, session extraordinaire 1991-1992, n 100/16-2º p. 10-14.

41 X. PACREAU, « Les conditions juridiques des opérations extérieures allemandes », A.F.R.I., vol. XIII, 2012, pp. 389-416.

42 C. Defrance, F. Knopper et A.-M. Saint-Gille (éds.), Pouvoir civil, pouvoir militaire en Allemagne, Aspects politiques, sociaux et culturels, Villeneuve d'Ascq, Presses Universitaires du Septentrion, 2013, p. 17.

43 Pour une excellente synthèse de ces débats doctrinaux jusqu'au début des années 1990, cf. C. TOMUSCHAT, «Les opérations des troupes allemandes à l'extérieur du territoire allemand », A.F.D.I., Vol. XXXIX, 1993, pp. 354-363.

${ }^{44}$ BVerfG, arrêt du 12 juillet 1994 (arrêt AWACS), BVerfGE 90, 286 ; Pour un commentaire de cet arrêt, voy. GöTZ SCHULZE et URS SAAL, «Les conditions d'engagement de la Bundeswehr dans des opérations militaires à l'étranger », in: Gratiae Fructus, Festschrift zu Ehren der Universität Lausanne, Regensburg, Donau-Druck, 1997, pp. 105-115.
} 
opérations de gestion de crise et de préventions de conflits ${ }^{45}$. Autrement dit, les opérations armées doivent être encadrées dans cette dernière hypothèse par un mandat de l'ONU ou par une décision de l'OTAN ou de l'Union de l'Europe occidentale, incorporée à l'Union européenne par le Traité de Lisbonne ${ }^{46}$. L'arrêt de la Cour constitutionnelle a été confirmé et approfondi à de nombreuses reprises ${ }^{47}$.

C'est ainsi qu'en 1995, la Bundeswehr a participé au déploiement de l'IFOR (Implementation Force) puis de la SFOR (Stabilisation Force) en Bosnie-Herzégovine. En 1998, elle a contribué à la « Force d'extraction » de Macédoine et, en 1999, des avions de combat allemands sont engagés au Kosovo dans l’opération « Forces alliées ». Le Bundestag vota également en novembre 2001 la confiance au gouvernement du chancelier Gerhard SCHRÖDER pour permettre l'envoi de soldats allemands en Afghanistan dans le cadre de l'opération Enduring Freedom. Encore récemment, en novembre 2015, à la suite des attentats perpétrés à Paris le 13 novembre 2015, l'Allemagne a pris la décision de déployer 1200 soldats pour participer à la lutte contre l'État islamique. Telle que conçue initialement, cette opération à l'étranger serait la plus vaste menée par le pays, selon le chef d'État-Major de la Bundeswehr.

La Loi fondamentale allemande fait du ministre de la Défense le chef des armées en temps de paix eu égard à son article $65 \mathrm{a}$. L'article $115 \mathrm{~b}$ prévoit cependant que ce rôle appartient au Chancelier en cas d'agression ou de menace imminente d'agression qui entraîneraient l'état de défense, constatée par le Bundestag, avec l'approbation du Bundesrat (article 115a), l'armée demeurant parlementaire (Parlamentsheer).

\footnotetext{
${ }^{45}$ Selon les termes exacts du Tribunal constitutionnel, « Die Beteiligung der Bundeswehr an den Aktionen von NATO, WEU und Vereinten Nationen ist nach der Entscheidung des Bundesverfassungsgerichts grundsätzlich mit dem Grundgesetz vereinbar. Sie findet ihre verfassungsrechtliche Grundlage in Art. 24 Abs. 2 GG, der mit der Ermächtigung zur Einordnung in ein System gegenseitiger kollektiver Sicherheit zugleich die Befugnis ausspricht, die Streitkräfte der Bundesrepublik bei allen Operationen einzusetzen, die im Rahmen und nach den Regeln dieses Systems stattfinden (aa). Um ein System gegenseitiger kollektiver Sicherheit im Sinne des Grundgesetzes handelt es sich nicht nur bei den Vereinten Nationen, sondern auch bei Systemen kollektiver Selbstverteidigung wie der NATO (bb). Art. 87a Abs. 2 GG steht Bundeswehreinsätzen, die sich auf Art. 24 Abs. 2 GG stützen lassen, von vornherein nicht entgegen (cc)...

Der parlamentarischen Zustimmung bedarf nach dieser Entscheidung auch die Unterstützung von Aktionen des Sicherheitsrates, und zwar unabhängig von der Ausgestaltung der Kommandostrukturen und unabhängig davon, ob den Streitkräften Zwangsbefugnisse nach Kapitel VII der Charta der Vereinten Nationen eingeräumt sind. Eine differenzierende Behandlung der verschiedenen Einsatzformen von Friedenstruppen verbiete sich schon deshalb, weil die Grenzen zwischen traditionellen Blauhelm-Einsätzen und militärischen Sanktionsmaßnahmen in der Realität fließend geworden seien ».

46 En ce qui concerne le cadre multilatéral des interventions de la Bundeswehr par rapport à la Loi fondamentale, voy. M. GReUBER, «Der Einsatz der Bundeswehr im Ausland : Die verfassungsrechtlichen Grenzen der Bündnispflicht », Grin, 2003, pp. 2-17.

47 BVerfG, 2 BvE 6, 99, 22 novembre 2001 ; BVerfG, 2 BvE 1/03, 7 mai 2008 ; BVerfG, 2 BvE 2/07, 3 juillet 2007 ; BVerfG, 2 BvE 2/08, 30 juin 2009; voy. UlRICH Fastenrath, "Anmerkung zum Urteil des Bundesverfassungsgerichts vom 3.7.2007 - 2 be 2/07 (Tornado-Einsatz in Afghanistan) », Juristenzeitung, vol. II, 2008, pp. 94-96.
} 
La décision d'envoyer des troupes nécessite donc, pour que l'intervention soit constitutionnelle (konstitutive Zustimmung) un vote du Bundestag en ce sens ${ }^{48}$, même quand l'opération fait suite à l'agression d'une nation alliée. Les modalités d'intervention sont fixées par le législateur. Cela ne porte pas atteinte au droit, pour le gouvernement, d'engager provisoirement les forces en cas de péril imminent (Gefahr im Verzug), notamment en application de décisions internationales ou de sauvetages, sans d'autorisation parlementaire préalable. Cela va de soi, il doit sans délai saisir le Bundestag pour confirmer l'opération en cours.

La participation de soldats allemands aux missions de surveillance par des aéronefs AWACS de l'OTAN a relancé le débat quant à l'opportunité d'adopter un cadre législatif aux opérations extérieures. La loi dite «de participation du Parlement» (Parlamentsbeteiligungsgesetz) a été adoptée en 2004 et promulguée le 18 mars 2005, après son adoption par le Bundesrat, pour consolider la jurisprudence constitutionnelle.

Les pouvoirs de l'exécutif restent importants ${ }^{49}$. Il a à lui seul le pouvoir d'enclencher une opération extérieure, selon les modalités qu'il détermine, et il ne peut y être contraint par le Bundestag. Certes, l'article 3 de la loi de participation du Parlement dispose que le gouvernement doit, dans sa demande d'autorisation, indiquer le théâtre d'opération, la base juridique de l'opération, le volume maximal des soldats et des forces mobilisés, la durée et le coût prévisibles de l'opération, mais le Bundestag ne peut qu'approuver ou repousser la motion en bloc. En outre, l'urgence permet de justifier une absence totale d'approbation parlementaire ${ }^{50}$.

Autre système parlementaire, le Japon est tout à fait particulier puisque l'article $9 \mathrm{du}$ chapitre II de la nouvelle Constitution, adoptée à la suite de la seconde guerre mondiale 51 , précise que, " [a]spirant sincèrement à une paix internationale fondée sur la justice et l'ordre, le peuple japonais renonce à jamais à la guerre en tant que droit souverain de la nation, ou à la menace, ou à l'usage de la force comme moyen de règlement des conflits internationaux.

\footnotetext{
${ }^{48}$ Article 42 (2) de la Loi fondamentale.

${ }^{49}$ M. DRAIN, «La dimension parlementaire de la politique de défense allemande », Les notes du Cerfa, 115, 2014, $52 \mathrm{p}$.

50 BVerfG, 2 BvE 6/11, 23 septembre, 2015.

${ }^{51}$ Le préambule de la Constitution dispose par ailleurs « Nous, le peuple japonais (...), décidés à ne jamais plus être les témoins des horreurs de la guerre du fait de l'action du gouvernement, proclamons que le pouvoir souverain appartient au peuple».
} 
Pour atteindre le but fixé au paragraphe précédent, il ne sera jamais maintenu de forces terrestres, navales et aériennes, ou autre potentiel de guerre. Le droit de belligérance de l'État ne sera pas reconnu $»^{52}$.

La nouvelle Constitution a enlevé beaucoup de pouvoir à l'Empereur, au bénéfice du Parlement et du Cabinet, puisque l'article 4.41 de la nouvelle Constitution dispose que: " [1]a Diète [la Chambre des représentants et la Chambre des conseillers] est l'organe suprême du pouvoir d'État, et le seul organe légiférant de l'État ».

En 1954, avec l'éclatement de la guerre de Corée, les Japonais se dotent malgré le dispositif constitutionnel d'une Force d'auto-défense ${ }^{53}$. Depuis cette époque où l'existence de ce corps militaire faisait déjà l'objet de controverse, celle-ci est sortie de son cadre étroit pour jouer un plus grand rôle au niveau international. Le Japon justifie l'envoi de ses troupes dans le cadre d'une vision strictement défensive et de protection des intérêts de l'État. Si déclarer la guerre est impossible, l'envoi de troupes à l'étranger doit être approuvé par le Parlement.

On remarquera à cet égard qu'à l'heure où ces lignes sont écrites, une procédure vivement contestée $^{54}$ de révision constitutionnelle est en cours au Japon dans l'optique d'amender l'article 9 et permettre l'adoption d'un projet de loi déposé par le gouvernement de M. Shinzo ABE sur la sécurité qui autorise les Forces d'autodéfense à participer à des opérations extérieures dans un certain nombre d'hypothèses. Cela s'inscrit dans la dynamique actuelle qui cumule la participation à des opérations internationales, certaines mandatées par l'ONU, la création d'un Ministère de la Défense en 2007 et la construction, en 2010, d'une base militaire à Djibouti.

La chose, en régime présidentiel, est nettement différente. Aux États-Unis, archétype d'un tel système, le Président, tel que le synthétisait par exemple Richard NixON, « est toutefois vital pour les questions de politique étrangère ${ }^{55}$. Effectivement, même si la Constitution de 1787, dans ses dispositions relatives à la politique étrangère et au partage des responsabilités entre le Président et le Congrès, reste vague ${ }^{56}$, elle rend possible la conduite de la politique extérieure et de défense en tenant à l'écart, dans une certaine mesure, le Congrès. Le président, d'après l'article II, section 2 , est « commandant en chef »

\footnotetext{
52 K. SchlichtmanN, « Artikel 9 im Normenkontext der Staatsverfassungen. Souveränitätsbeschränkung und Kriegsverhütung im 20. Jahrhundert», in: W. ShaumanN (éd.), Gewollt oder geworden? - Planung, Zufall, natürliche Entwicklung in Japan; Referate des 4. Japanologentags der OAG in Tokyo, 17/18. März 1994, Munich, Iudicium, 1996, pp. 129-150.

${ }^{53}$ J. E. AuER, « Article Nine of Japan's Constitution: From Renunciation of Armed Force 'Forever' to the Third Largest Defense Budget in the World », Law and Contemporary Problems, 1990, pp. 171-187.

54 «Japan security bills reveal irreconciliable divide between scholars, politicians », The Japan Times, 12 juin 2015.

55 J. Yochelson, «Le débat aux États-Unis sur la présence et l'engagement militaire en Europe », Politique étrangère, 1971, p. 836.

56 M.-F. ToINET, «Les difficultés intérieures de la politique étrangère américaine», Défense nationale, janvier 1979, p. 68.
} 
des armées ${ }^{57}$, tandis que le Congrès, selon l'article I, section 8 , « déclare la guerre » et « lève et entretient les armées ${ }^{58}$. Par ailleurs, le Congrès a déjà cherché à juguler davantage le pouvoir de l'exécutif par la voie du budget ${ }^{59}$. Le Congrès a déjà contesté la monopolisation présidentielle qu'il a estimée contraire à la Constitution dans la foulée de la guerre du Vietnam et surtout de l'intervention au Cambodge.

Deux visions d'opposent en conséquence ; la première, proche de l'enseignement de l'arrêt United States v. Belmont ${ }^{60}$ veut que le président dispose de "pouvoirs inhérents " en matière étrangère tandis que la seconde dispose que c'est au Congrès de fixer la politique, étant entendu que l'intervention du Congrès est exigée par la Constitution ${ }^{61}$.

Cela étant, au fur et à mesure du $\mathrm{XX}^{\mathrm{e}}$ siècle, et principalement le déclenchement de la seconde guerre mondiale, le Président est de plus en plus devenu le seul maître de la politique étrangère, avec le Département d'État en support. L'exécutif est devenu le seul pouvoir capable d'en préparer les données et d'en suivre la mise en œuvre, et ce même s'il n'existe pas de menaces imminentes pour les États-Unis. Quelques accrocs ont toutefois surgi au Congrès dès les années 50 , sous la présidence du Général EISENHOWER lorsque les sénateurs BRICKER et KNOWLAND, cherchèrent à restaurer, par voie d'amendement à la Constitution, les prérogatives du pouvoir législatif dans le domaine des accords internationaux $^{62}$. Le Congrès, néanmoins, n'a pas sérieusement contesté la prééminence présidentielle clairement revendiquée et exercée par de nombreux présidents ${ }^{63}$.

Le traumatisme du Vietnam a bousculé cette vision presque d'infaillibilité présidentielle ${ }^{64}$. L'invasion du Cambodge au début de 1970, sans que le Congrès eût été consulté ni même averti, a marqué les esprits du pouvoir législatif. Le 7 novembre 1973, le Congrès a adopté définitivement le War Powers Resolution Act malgré le véto présidentiel de Richard NixoN65. Cette Loi sur les pouvoirs de guerre dispose notamment que le chef de l'Exécutif doit faire rapport au Congrès dans les 48 heures d'un engagement des troupes à l'étranger et impose un délai de 60 jours au terme duquel le Président, sauf autorisation du Congrès, doit interrompre les opérations militaires. Dans ce dernier délai, il est possible pour les deux assemblées de demander, par "résolutions concurrentes", la cessation immédiate de

\footnotetext{
57 P. BARRAL, Pouvoir civil et commandement militaire : du roi connétable aux leaders du 20e siècle, Paris, Presses de Science Po, 2005, pp. 96-99.

${ }^{58}$ D. P. Currie, The Constitution of the United States: A Primer for the People, 2e éd., Chicago et Londres, The University of Chicago Press, 2010, p. 40.

59 J. YOCHELSON, op. cit., p. 7.

60301 U.S. 324, 1937.

${ }^{61}$ P. VIALLE, « Le Congrès, le Président et la politique étrangère », Revue internationale de droit comparé, 1979, p. 603.

62 S. GARRET, «Foreign Policy and the American Constitution : The Bricker Amendment in Contemporary Perspective », International Studies Quarterly, 1972, p. 187.

63 P. VIALLE, op. cit., p. 604.

${ }^{64}$ H. Bradford WeSterfield, « Congress and Closed Politics in National Security Affairs », Orbis, 1966, pp. 740753.

65284 voix contre 135 à la Chambre, 75 voix contre 18 au Sénat.
} 
l'action armée américaine. C'est ainsi qu'en avril 1975, alors que le Vietnam du Sud tombait, le Président FORD a souligné que, malheureusement, le Congrès lui a, en 1973, retiré le pouvoir d'utiliser des moyens militaires pour faire respecter les accords signés à Paris ${ }^{66}$. L'effectivité concrète de cette norme législative est cependant pour le moins contestable : moins de deux ans après l'adoption du War Powers Act, le même Président Gerald FORD choisit, à la suite de la capture du porte-conteneurs civil américain $S S$ Mayagüez par les forces Khmers rouges au large des côtes du Cambodge, d'engager des forces militaires pour secourir des citoyens américains en l'absence de toute consultation du Congrès qui, dans sa grande majorité se contenta d'approuver l'initiative ${ }^{67}$. Lorsque le Président Bill Clinton a ordonné, en 1999, le bombardement au Kosovo, la loi n’a pas plus été respectée. De même, le 20 mai 2011, au soixantième jour d'engagement des troupes américaines en Libye, le Président Barack OBAMA a notifié qu'aucune autorisation du Congrès n'est requise dès lors que l'opération - limitée en ses termes - est à ce moment dirigée par l'OTAN. Conscient du caractère fragile de ce tigre de papier, certains sénateurs ont proposé en 2014 de remplacer cette loi par une norme plus en phase avec la fréquence des conflits locaux afin de n'exiger la consultation préalable du Congrès que pour les cas d'engagement militaire important. On peut facilement et synthétiquement constater que la loi n'a pas inversé l'équilibre qui, historiquement et politiquement, fait du Président des États-Unis l'acteur prédominant en matière militaire, et, en temps de guerre formellement constatée par le Congrès, le Président reçoit de véritables pouvoirs exceptionnels dans ces circonstances ${ }^{68}$.

Dans un régime semi-présidentiel, la chose est encore différente.

En France, l'article 5, alinéa 2, de la Constitution de 1958 fait du Président de la République «le garant de l'indépendance nationale, de l'intégrité du territoire et du respect des traités » ${ }^{69}$. L'article 13, alinéa 2, dispose quant à lui que « [le Président] nomme aux emplois civils et militaires ». Enfin, et surtout, l'article 15 de la Constitution dispose que le Président de la République est le chef des armées ${ }^{70}$. Il préside les conseils et comités supérieurs de la défense. A plusieurs égards, la pratique de la Ve république va fondamentalement modifier les rapports du Chef de l'État avec les armées.

La politique de défense est définie par le Chef de l'État qui, à la différence de ses prédécesseurs de la IVe République, jouit de prérogatives concrètes, notamment la

\footnotetext{
66 Déclaration du Président Gerald FoRD du 21 avril 1975, citée par F. R. BAX, «The Legislative-executive Relationship in Foreign Policy : New Partnership or New Competition », Orbis, 1977, p. 882, note 3.

${ }^{67}$ New York Times, 23 mai 1975, p. 21.

68 Voy. D. L. WeSTERFIELD, War powers - The president, the congress, and the Question of War, Wesport, Praeger, 1996, 245 p.; M. ZeISBerg, War Powers: The Politics of Constitutional Authority, Princeton, Princeton University press, 2013, $288 \mathrm{p}$.

69 B. Chantebout, "Constitution et Défense nationale», Droit et défense, 1999, pp. 5-9 ; S. CoHEN, «Le Président, chef des armées », Pouvoirs, n58, 1991, pp.33-40 ; M.-T. VIEL, «La répartition des compétences en matière militaire entre le Parlement, le Président de la République et le Premier ministre », R.D.P., 1993, pp.141-195.

${ }^{70}$ F. FISSON-Roche, op. cit., pp. 504-524.
} 
présidence des conseils de défense. Or, le Président de la Ve République «tient sa suprématie en cette matière, autant de la nature des textes que d'une pratique bien enracinée qui font de lui à la fois l'ultime recours et le maître d'œuvre de la défense ». L'ordonnance du 7 janvier 1959 nouvellement codifiée conforte implicitement la position présidentielle. L'article L. 1111-3 du Code de la défense précise que « [l]a politique de la défense est définie en conseil des ministres. Les décisions en matière de direction générale de la défense sont arrêtées en comité de défense. Les décisions en matière de direction militaire de la défense sont arrêtées en comité de défense restreint », ces différents organes collégiaux étant présidés eux-mêmes par le Président de la République. Ainsi, le Président Nicolas SARKOZY ne faisait que suivre la pratique constitutionnelle en annonçant, en mars 2008 l'envoi de nouvelles troupes en Afghanistan, et ce, sans vote parlementaire. Le Président François HoLLANDE ne remet pas non plus en cause cette pratique institutionnelle en prenant la décision en 2013 de mener une opération de libération d'un membre de la DGSE en Somalie et, depuis ce moment, de lancer plusieurs opérations militaires d'envergure, notamment les opérations Serval et Chammal, afin d'arrêter la propagation islamiste, respectivement au Mali et dans une portion de territoire qui couvre une partie de l'Irak et de la Syrie.

Les articles 20 et 21 de la Constitution du 4 octobre 1958 confient au gouvernement, responsable devant le Parlement, la détermination et la conduite de la politique de la nation et au Premier ministre la direction de son action. Aucune décision positive ne peut donc être prise sans l'accord du président et du gouvernement. Le Président de la République et le Premier ministre sont tous les deux dépositaires de compétences, mais la pratique révèle la primauté présidentielle, bien qu'une concurrence au sein du pouvoir exécutif ne soit pas impossible. Le général DE GAULLE a défendu la présidentialisation du pouvoir militaire afin que « désormais, le chef de l'État soit réellement le chef des armées, bref qu'émanent de lui toute décision importante aussi bien que toute autorité ${ }^{71}$, le premier ministre en tant qu'autorité administrative n'agissant que sur la base des directives du Chef de l'État. Hormis l'aspect strictement opérationnel, les différents aspects $\mathrm{du}$ pouvoir militaire reviennent tout de même largement au Premier ministre et ses compétences réglementaires font de lui un acteur indispensable: toutes les décisions réglementaires - à l'exception de celles prévues par la Constitution à l'article 19 - doivent être contresignées par le chef du Gouvernement conformément à l'article 13 de la Constitution. Dans le cas qui nous intéresse, cela concerne bien toutes les décisions prises en Conseil des ministres où la politique de défense, qui recouvre les lois de programmation militaire, les lois budgétaires annuelles, est arrêtée.

Ainsi, l'organisation des opérations extérieures relève de la double décision des autorités exécutives françaises. Dans toutes les crises, le Chef de l'État s'adresse à la Nation tandis le Premier ministre communique avec la Représentation nationale. Les ministres de la défense jouent un rôle particulier dans cette structure. Le ministre de la défense est le trait d'union entre les armées et le Président et le gouvernement. Il a pour mission de faire

${ }^{71}$ Ch. DE GAULle, Mémoires d'espoir. Le Renouveau : 1958-1962, Paris, Plon, 1970, p. 35. 
accepter par les militaires les orientations gouvernementales et transmettre les besoins et les revendications de l'armée ${ }^{72}$.

Depuis la révision constitutionnelle du 23 juillet 2008, l'Assemblée nationale - qui doit depuis le début de la Ve République autoriser la déclaration de guerre, jouit de pouvoirs supplémentaires en la matière puisque le pouvoir d'intervention des forces armées à l'étranger est davantage encadré. Le Gouvernement informe le Parlement au plus tard trois jours après le début d'une opération extérieure et, au delà d'un délai de quatre mois, sa prolongation est soumise à l'autorisation de ce dernier, selon l'article 35, al. 2 et 3, de la Constitution. L'alinéa 3 de l'article 35 a été utilisé pour la première fois le 22 septembre 2008 lorsqu'il a été question de prolonger l'intervention française en Afghanistan. Depuis lors, le pouvoir législatif a systématiquement autorisé la poursuite des opérations dirigées par l'exécutif. On observera en outre que, s'il est vrai que le Gouvernement peut être limité par le Parlement après un certain délai, la Constitution ne dit cependant rien en cas de prolongation de l'opération sur plusieurs années.

\section{B. La question de la responsabilité}

Sur le plan interne, la question est de savoir si un particulier, ou une association, a la possibilité de s'opposer au recours à la force mobilisé par le gouvernant. Or, la question de savoir si l'envoi des troupes à l'étranger est susceptible de recours nous amène à examiner la relative immunité de certains actes administratifs et, par conséquent, un concept mis en avant par le Doyen FAVOREU ${ }^{73}$ : les actes de gouvernement. L'acte de gouvernement est presque aussi ancien que la juridiction administrative.

On remarque à cet égard une forme d'uniformité des pratiques.

Dans son arrêt El-Shifa Pharm. Indus. co v. United States ${ }^{74}$, la Cour d'appel du Circuit fédéral des États-Unis a mis en avant la nature politique de l'engagement militaire, en précisant que : "where the President's own assessment of the offensive posture of the Nation's enemies overseas leads him to conclude that the Nation is at risk of imminent attack, we cannot find in the Constitution any support for judicial supervision over the process ». Le pouvoir de déterminer rétroactivement si l'intérêt des États-Unis a été d'utiliser les forces armées n'appartient dès lors pas aux juridictions, étant entendu que l'engagement militaire

\footnotetext{
72 S. COHEN, « Le pouvoir politique et l'armée », Pouvoirs, 2/2008, p. 24.

${ }^{73}$ L. FAVOREU, Du déni de justice en droit public français, Paris, LGDJ, 1964, 582 p. ; voy. également L. FAVOREU, "L'acte de Gouvernement, acte provisoirement et accidentellement injusticiable », R.F.D.A., 1987 p. 544 et s. ; F. MELLERAY, «L'immunité juridictionnelle des actes de gouvernement en question », R.F.D.A., 2001, p. 1086 et s. ; L. FAVOREU, "Pour en finir avec la "théorie" des actes de Gouvernement », in : Mélanges en l'honneur de Pierre Pactet, Paris, Dalloz, 2003, p. 607 et s.; E. CARPEnTIER, " L' “acte de Gouvernement” n'est pas insaisissable », R.F.D.A., 2006 p. 661 et s. ; E. CARPENTIER, "Permanence et unité de la notion d'acte de Gouvernement », A.J.D.A., 2015 p. 799 et s.

${ }^{74}$ El-Shifa Pharm. Indus. v. United States, 378 F.3d 1346 (Fed. Cir. 2004).
} 
ressort du domaine du Congrès et de l'Exécutif selon l'arrêt Baker v. Carr $^{75}$ de 1962. L'évolution de la théorie de la question politique ${ }^{76}$, depuis l'arrêt Marbury v. Madison au sein duquel le Chief Justice John MARSHALL a reconnu que "some discretionary actions by other branches lie outside the scope of judicial review ${ }^{77}$, fait désormais du Congrès le contre-pouvoir à l'action gouvernementale en matière de politique étrangère et, à plus forte raison, des prérogatives militaires ${ }^{78}$.

En Belgique, une telle théorie n'est pas clairement consacrée et l'existence de la notion d'actes de gouvernement reste encore controversée ${ }^{79}$. Au cours des travaux préparatoires de la loi du 23 décembre 1946 portant création du Conseil d'État ${ }^{80}$, un compromis s'est dégagé lors des débats en commission pour exclure du contrôle de la juridiction administrative les «actes de gouvernement» au rang duquel se retrouve le commandement des forces armées ${ }^{81}$. Cependant, la jurisprudence subséquente du Conseil d'État demeure fortement ambigüe ${ }^{82}$.

En France, fait également écho à la théorie de l'acte de gouvernement la décision Mégret et Mekhantar ${ }^{83}$ de 2000 par laquelle le Conseil d'État s'est déclaré sans compétence pour connaître du recours visant la décision du Président de la République d'engager un corps de l'armée française dans les opérations de l'OTAN contre la République fédérale de Yougoslavie à la suite de la crise du Kosovo. De même, en 2003, le Conseil d'État conclut à l'incompétence de la juridiction administrative pour connaître du référé-suspension concernant l'autorisation par la France du survol du territoire par les armées de la coalition dirigée contre l'Irak et le rejette en réemployant la formule de l'arrêt Mégret et Mekhantar et en précisant que « la décision des autorités françaises d'autoriser les avions militaires américains et britanniques qui accomplissent des missions en Irak à emprunter l'espace aérien français [...] n'est pas détachable de la conduite des relations internationales de la France ${ }^{84}$. Traditionnellement, donc, il est admis que tout acte administratif qui met

\footnotetext{
75 Baker v. Carr, 369 U.S. 186 (1962) ; voy. également Rappenecker v. United States, 509, F.Supp. 1024 (N.D. Cal. 1980) ; Conyen v. Reagan, 578, F.Supp. 324 (D.D.C. 1984) ; Lowry v. Reagan, 676, F.Supp. 333 (D.D.C. 1987), aff'd, No 87-5426 (D.C. Cir. 1988) ; Doe v. Buch, 240, F.Supp. 2d 95 (D. Mass. 2002).

76 E. B. FIRMAGE, «The war powers and the political question doctrine », University of Colorado Law Review, 1977, pp. 65-101 ; N. MourtadA-SABBAH et B. E. CAIn, The Political Question Doctrine and the Supreme Court of the United States, Plymouth, Lexington Books, 2007, 273 p.

77 Marbury v. madison, 5 US (1 CR), 137, 164, (1803).

${ }^{78}$ L. FISHER, « Judicial Review of the war power », Presidential Studies Quarterly, 35, n³, 2005, pp. 466-495.

79 J. Velaers, « De Grondwet en de krijgsmacht», in : M. VAN DAmme, L. DE Vos, J. Velaers, L. F.M. BesSELINK, E. VANDENBOSSCHE et G. VERHOFSTADT, De grondwet het inzetten van strijdkrachten, Anvers, Maklu, 2005, p. 121.

${ }^{80}$ Moniteur belge, 7 janvier 1947.

${ }^{81}$ Doc. parl., Sénat de Belgique, session extraordinaire, 1939, n 80, pp. 26 et 43.

82 Voy. C. Huberlant, « Les actes de gouvernement », Revue de droit international et de droit comparé, 1954, pp. 282 et s. ; R. Ergec, « Le contrôle juridictionnel de l'administration dans les matières qui se rattachent aux rapports internationaux : actes de gouvernement ou réserve du pouvoir discrétionnaire», Revue de droit international et de droit comparé, 1986, pp. 72-134.

${ }^{83}$ CE fr., 5 juillet 2000, Mégret et Mekhantar, A.J.D.A, 2001, p. 95.

84 C.E. fr., ord. 10 avril 2003, Comité contre la Guerre en Irak, A.J.D.A., 2004, p. 613 et également C.E. fr, 30 décembre 2003, Comité contre la guerre en Irak et autres, ${ }^{\circ} 255.904$, R.T.D.H., n64, p. 855 et s., obs C. RAUX.
} 
directement en œuvre la politique étrangère, la défense et la sécurité nationale ne puisse en principe faire l'objet d'un recours en justice.

Cela ne préjuge rien au fait que de nombreux États maintiennent une compétence juridictionnelle - en règle générale au sein de la structure militaire - pour connaître de certains actes posés par les forces au sol.

Il reste encore à dépasser les frontières internes et aborder la question du cadre juridique au niveau international. Certes, contrairement à d'autres domaines, la défense, compétence régalienne de l'État, n'a subi que peu d'érosion de la part de la communauté internationale. Compte tenu du caractère intergouvernemental des processus de décision dans les organisations multilatérales (ONU, OTAN, OSCE et UE), le gouvernement reste souverain et maître de sa contribution : si le pouvoir d'engagement reste dans le giron national, il n'est jamais automatique. Après tout, c'est précisément parce que la responsabilité des pertes humaines subies par une force militaire demeure à la charge de l'État que celui-ci est susceptible de remettre en cause sa participation au sein des structures supranationales. L'article 31 du TUE (ex-article 23 TUE) est à ce titre sans équivoque; le Conseil des ministres de l'Union européenne vote à l'unanimité pour des décisions ayant des implications militaires ou dans le domaine de la défense. Le pouvoir d'engagement reste clairement national.

Pourtant, la guerre, somme toute, est conditionnée par le droit international public. On remarquera à ce titre que le Préambule de la Constitution de 1946 reconnaît que « [1]a République française, fidèle à ses traditions, se conforme aux règles du droit public international. Elle n'entreprendra aucune guerre dans des vues de conquête et n'emploiera jamais ses forces contre la liberté d'aucun peuple».

En droit international, il convient de distinguer le droit ad bellum du droit in bello, c'est-àdire Le droit de faire la guerre et le droit dans la guerre, appelé également droit international humanitaire. Or, la société internationale a posé pour principe fondateur l'égalité juridique entre les États selon l'article $2, \S 1^{\mathrm{er}}$, de la Charte des Nations Unies et a formellement interdit l'emploi de la guerre (ius ad bellum), sans préjudice des mesures ordonnées par le Conseil de sécurité et la légitime défense. En d'autres termes, il existe une obligation, consacrée par la Charte des Nations-Unies et la coutume internationale, de ne pas faire la guerre. Si, auparavant, la guerre n'était qu'une manifestation classique de la souveraineté des États, le principe du non-recours à la force a pour origine le pacte de la Société des Nations qui avait réduit, suite à la première guerre mondiale, le pouvoir discrétionnaire de l'État à faire la guerre. Mais c'est par le pacte Briand-Kellog, conclu le 26 août 1928, que le principe d'une interdiction de la guerre a été adopté - seulement entre hautes parties contractantes, il est vrai.

Le ius ad bellum s'est ainsi vu radicalement transformé. Après la seconde guerre mondiale, le principe est renforcé par la création de l'Organisation des Nations Unies (ONU). Le principe de l'interdiction du recours à la force est une pièce maîtresse dans l'édifice du système de sécurité collective mis en place au lendemain de la seconde guerre mondiale et rappelée à de nombreuses reprises par les résolutions de l'Assemblée générale des Nations 
Unies. En 1986, la Cour internationale de Justice a constaté dans l'arrêt Activités militaires

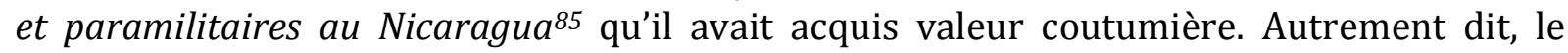
principe de l'interdiction du recours à la force a donc le double statut de norme conventionnelle et de norme coutumière. Il est par conséquent véritablement universel. Enfin, eu égard à son importance, cette norme est souvent citée dans la littérature du droit international public comme une illustration des normes de ius cogens, insusceptibles de dérogation ${ }^{86}$.

La guerre, classiquement, c'est l'invasion d'un État par un autre État. En pratique, cela dit, les guerres ne sont plus déclarées. Même si la France n'a jamais déclaré la guerre depuis la seconde guerre mondiale, elle déploie en 2013 près de 12000 militaires dans des opérations militaires menées sur des sols étrangers. Dans cette perspective, on préfère en effet parler de force et d'opération extérieures pour désigner toutes les opérations militaires et para- militaires. Par conséquent, vu l'interdiction contemporaine du recours à la force, les différents États permettent de moins en moins au Chef de l'État de « déclarer la guerre », mais seulement d'en " constater l'état». Ainsi, en Belgique, le texte de l'actuel article 167 indique que le Roi «constate l'état de guerre», mais ne la déclare pas. Si le concept de guerre n'a pas changé, c'est le rôle défensif que la Belgique, à l'instar de bien d'autres, entend se donner dans le déclenchement des hostilités qui est modifié. De nos jours, les États privilégient les expressions « opération militaire », « opération de maintien de la paix » ou « mission militaire » qui évitent le mot à consonance bien négative. Les États ont voulu ainsi s'affranchir symboliquement de l'incompatibilité évidente entre la Charte des Nations Unies et leurs pratiques extérieures. Cela n'empêche pas, pour autant, le risque d'engager sa responsabilité internationale si l'un des États se risque à une expédition armée sans trouver de justifications juridiques adéquates.

Visant les dirigeants et les haut-gradés des forces armées, la notion de crime d'agression, ou de crime contre la paix, est centrale puisque elle a été définie dans le cadre des Tribunaux militaires internationaux de Nuremberg et de Tokyo établis après la Seconde Guerre mondiale comme «la direction, la préparation, le déclenchement ou la poursuite d'une guerre d'agression, ou d'une guerre en violation des traités, assurances ou accords internationaux, ou la participation à un plan concerté ou à un complot pour l'accomplissement de l'un quelconque des actes qui précèdent ${ }^{87}$. En 2010, à la suite de la Conférence de Kampala pour réviser le Statut de Rome de la Cour pénale internationale, un article 8bis a été ajouté au Statut de Rome pour définir le crime d'agression.

En matière de droit in bello, on remarquera également que le Chef de l'État, devenu en quelque sorte destinataire de normes de droit international public, peut se rendre

\footnotetext{
85 Activités militaires et paramilitaires au Nicaragua et contre celui-ci (Nicaragua c. Etats-Unis d'Amérique), fond, arrêt. C.I.J., Recueil, 1986, p. 14.

${ }^{86}$ Voy. sur ce sujet O. CORTEN, The law against war - The prohibition on the Use of Force in Contemporary International Law, Oxford and Portland, Hart Publishing, 2012, 569 p.

87 « Confirmation des principes de droit international pénal reconnus par les Tribunaux Militaires Internationaux », Résolution 95 (I) de l’Assemblée générale des Nations unies adoptée le 11 décembre 1946.
} 
techniquement coupable de crimes de guerre. D'ailleurs, seule une personne physique peut être condamnée pour un tel crime. Le principe de la responsabilité individuelle des auteurs d'un crime de guerre se retrouve dans les statuts du Tribunal pénal international pour l'exYougoslavie (à l'article 7), du Tribunal pénal international pour le Rwanda (à l'article 6) et de la Cour pénale internationale (à l'article 25) ainsi que dans le projet de code des crimes contre la paix et la sécurité de l'humanité de la Commission de droit international (à l'article 2). Dans l'affaire Ohlendorf (Einsatzgruppen trial), le tribunal militaire américain de Nuremberg a pu juger que: "Nations can only act through human beings, and when Germany signed, ratified and promulgated the Hague and Geneva Conventions, she bound each one of her subjects to their observance [...]. The defendants are in court not as members of a defeated nation but because they are charged with crime $\$ 88$. Or, il ne faudrait guère croire que le ius in bello ne s'applique qu'au conflit officiellement nommé "guerre »; au contraire, l'on constate que le droit des conflits armés s'applique à d'autres types de conflits armés :

- le conflit armé international considéré de manière extrêmement large et souple ;

- le conflit armé non international qui vise une guerre civile classique opposant un gouvernement à des insurgés qui contrôlent de manière continue une partie de territoire (comme en Géorgie, au Rwanda, en Somalie ou en Syrie).

Élément supplémentaire du monde moderne, l'émergence des organisations régionales de protection des droits fondamentaux fixe de nouvelles bornes à l'action du Chef de l'État. Que ce soit lors des opérations anti-terroristes ou anti-piraterie, les États sont aux prises avec deux logiques en apparente contradiction : «la logique d'efficacité de l'exécutif et la logique d'efficacité du judiciaire, qui doit préserver l'État de droit des risques d'excès sécuritaires ». La Cour européenne des droits de l'homme a pu affirmer, le 12 juillet 2008, dans l'affaire Medvedyev c. France ${ }^{89}$, à propos de la lutte contre le trafic de stupéfiants qu' « aussi légitime qu'elle soit, une telle fin ne saurait justifier tous les moyens ». Les États sont tenus de garantir à toute personne relevant de leur juridiction les droits et libertés garantis par les instruments conventionnels de protection des droits de l'homme qu'ils ont ratifiés, en toute circonstance et dans les seules limites prévues par ces textes. En particulier, la Convention européenne des droits de l'homme «ne s'arrête pas aux portes des casernes ».

La jurisprudence européenne présente plusieurs contentieux ayant trait à des conflits armés, tant sur le territoire européen qu'au-delà de ses frontières ${ }^{90}$. Ainsi, dans l'affaire $\mathrm{Al}$ -

\footnotetext{
886 trials of War Criminals Before the Nuernberg Military Tribunal Under Control Council Law No, 10462 ('Einsatzgruppen Case')

${ }^{89}$ Cour eur. dr. h., arrêt Medvedyev c. France, 10 juillet 2008.

90 On peut ainsi lire dans l'arrêt Cour eur. dr. h., arrêt Al-Saadoon et Mufdhi c. Royaume-Uni, 3 juillet 2009, § 85, que « when, as a consequence of lawful or unlawful military action, a Contracting State exercises effective control of an area outside its national territory, there may be an obligation under Article 1 to secure the Convention rights and freedoms within that area ».
} 
Skeini et autres, la Grande Chambre - à l'unanimité de ses dix-sept juges - a condamné le Royaume-Uni pour violation du droit à la vie consacré à l'article 2 en son volet procédural ${ }^{91}$. Aux yeux de la Cour, les autorités de cet État ont manqué à leur obligation d'enquête effective sur le décès de plusieurs civils irakiens en 2003 dans lesquels étaient impliqués des soldats britanniques. Dans l'affaire Al-Jedda, le Royaume-Uni a été condamné pour violation du droit à la liberté et à la sûreté, consacré par l'article 5 de la Convention européenne des droits de l'homme, au motif que les autorités du Royaume-Uni ont emprisonné un civil irakien pendant plus de trois ans dans un camp à Bassorah ${ }^{92}$. L'on assiste ainsi à l'extension territoriale des obligations conventionnelles, y compris en cas d'opérations militaires ${ }^{93}$.

\footnotetext{
${ }^{91}$ Cour eur. dr. h., arrêt Al-Skeini et autres c. Royaume-Uni, 7 juillet 2011.

92 Cour eur. dr. h., arrêt Al-Jedda c. Royaume-Uni, 7 juillet 2011 ; voy. également Cour eur. Dr. H., arrêt Hassan c. Royaume-Uni, 16 septembre 2014.

93 Voy. également Court of Appeal of England and Wales, arrêt Serdar Mohamed c. Secretary of State, EWCA Civ., 840, 30 juillet 2015.
} 


\section{$2^{\mathrm{e}}$ Partie : Le Chef de l'État face au pouvoir militaire}

L'examen des interactions entre le pouvoir exécutif et les principes démocratiques opéré, il importe d'exposer un peu plus ce pouvoir puisque, nous l'avons vu, la volonté de gouverner les hommes ou d'orienter la politique en s'appuyant sur la force militaire s'observe en de nombreuses sociétés. Or, si pouvoirs civils et militaires se sont souvent confondus, désormais, tel n'est plus le cas. L'armée est subordonnée au chef de l'État dans l'ensemble des pays démocratiques occidentaux. Le chef de l'État représente ainsi la nation et le pouvoir civil qui en émane, ce qui n'est pas sans entraîner de nombreuses difficultés, y compris bureaucratiques ${ }^{94}$.

Toutefois, comme pour nombre d'États ayant un régime parlementaire dans le cadre de monarchies constitutionnelles (Grande- Bretagne, Belgique, Espagne) ou non (Allemagne, Autriche), le chef du Gouvernement est l'autorité politique réelle de l'armée. Dans ces régimes, si le chef de l'État symbolise l'État, le chef du Gouvernement constitue le pouvoir politique décisionnel et exécutif. Ainsi, la soumission de l'armée au pouvoir civil doit être commentée.

La soumission est symbolique avec le Chef de l'État, mais effective et démocratique avec le chef du Gouvernement. L'autorité politique - chef de l'État ou chef du Gouvernement - ne commande pas nécessairement opérationnellement l'armée mais elle en ordonne l'emploi. Cette distinction est nouvelle puisque, sous l'Ancien Régime, le Roi est un militaire et mène l'armée à la guerre. On remarque d'ailleurs que dans les monarchies, le lien entre royauté et armée est maintenu par l'éducation donnée au souverain. Outre une formation académique et politique, une formation militaire est dispensée à l'héritier du trône en Belgique et au Royaume Uni, à l'inverse des autres acteurs politiques dans ces États. Pourtant, l'évolution de la démocratisation de la société va démilitariser le pouvoir sur l'armée. Pour reprendre SAINT-JuST, « la direction du pouvoir militaire (je ne dis pas l'exécution militaire) est inaliénable de la puissance législative du souverain [le peuple] »95. Le chef militaire est donc un militaire, soumis à la volonté politique qui ne l'est pas.

Pour Samuel P. Huntington, dans son fameux ouvrage de 1957, The Soldier and the State: The Theory and Politics of Civil-Military Relations ${ }^{96}$, devenu un classique en la matière, le «professionnalisme militaire» est une garantie à la suprématie civile. Pour l'auteur, la montée en puissance du « militarisme » au sein des sociétés occidentales va provoquer une

\footnotetext{
94 Sur un aperçu des problématiques actuelles, voy. B. IRONDELLE, « Démocratie, relations civilo-militaires et efficacité militaire », Revue internationale de politique comparée, 2008, pp. 117-131.

${ }^{95}$ Cité par P. BARRAL, op. cit., p. 55

96 S. Huntington, The Soldier and the State: The Theory and Politics of Civil-Military Relations, New York, Belknap Press, 1957, 534 p.
} 
réaction de l'État pour tenter de réduire ou de neutraliser les menaces exercées par des forces armées de l'extérieur. Une nouvelle composante va ainsi se développer au sein des armées: le corps des officiers, autrement dit des professionnels de l'armée qui constitueraient la meilleure garantie du maintien du pouvoir civil. « L'auteur », synthétisent Joana JEAN et Marc SMYRL, «faisait de leur soumission au pouvoir politique un élément central du professionnalisme des militaires, considérant que la mise en place d'un contrôle « objectif » des forces armées, respectueux de leur sphère d'expertise propre, était le meilleur moyen d'assurer un équilibre de leurs rapports avec les gouvernants ${ }^{97}$.

Les rapports entre le pouvoir politique et l'armée ont longtemps été régis selon des règles simples, mais nécessaires: apolitisme et subordination, complète et inconditionnelle, gouvernement légal, compétent exclusivement de fixer les règles de recrutement, de fonctionnement et d'engagement, car l'unique rôle de la force armée est de permettre à celui-ci de garantir l'ordre public intérieur et de défendre le pays contre l'ennemi de l'extérieur.

Parmi les constitutions écrites, celle des États-Unis offre un grand nombre de précautions pour assurer la suprématie du pouvoir civil sur l'armée. Il faut dire que l'État a été créé dans un climat de méfiance et de scepticisme à l'égard de l'appareil militaire. Preuve s'il en faut de cet état d'esprit, le 2 juin 1784, le Congrès continental, convaincu par le discours d'Elbridge GERRY selon lequel «standing armies in time of peace are inconsistent with the principles of republican Governements, dangerous to the liberties of a free people, and generally converted into destructive engines for establishing despotism » a voté une résolution suivant laquelle: "Resolved that the commanding officer be and he is hereby directed to discharge the troops now in the service of the United States, except twenty-five privates, to guard the stores at fort Pitt, and fifty-five to guard the stores at West Point and other magazines, with a proportionate number of officers; no officer to remain in service above the rank of captain ${ }^{98}$.

La chose n'est pas restée en l'état, comme on le sait. Le Président dispose de hautes prérogatives militaires, et l'activité des autorités militaires sur le terrain est soumise au contrôle des tribunaux ordinaires. En outre, un trait symbolique traduit parfaitement la subordination des armées ; nous pensons en l'espèce au serment du soldat qui jure fidélité aux autorités civiles ${ }^{99}$ dont les ordres lui sont obligatoires. Après la seconde guerre mondiale, la guerre froide a relancé les débats relatifs aux relations entre le pouvoir exécutif et son armée. C'est au cours de cette période que les réflexions de Samuel HunTINGTON ont fortement nourri les discussions. Par ailleurs, les relations entre armée et président n'ont pas toujours été les plus sereines et l'un des bras de fer les plus intenses et les plus notoires a été celui, durant la guerre de Corée, entre Douglas MACARThuR, qui a en partie provoqué l'entrée de la Chine dans la guerre en contrevenant aux ordres, et le

${ }_{97}$ J. JOANA et M. SMYRL, « Civils et militaires en démocratie », Revue internationale de politique comparée, 2008, p. 7.

98 ForD et al., Journal of the Continental Congress, 25:715.

99 J. MEYNAUD, « Les militaires et le pouvoir », Revue française de sociologie, 1961, pp. 76-77. 
Président TRuman. Soutenant la présidence, le Joint Chiefs of Staff a admis l'écartement du héros de la seconde guerre mondiale, non sans déplorer les «interférences civiles » dans la conduite des opérations. Cela n'est pas isolé dans l'Histoire américaine; et, il est communément reconnu que les relations civilo-militaires pendant l'ère Clinton ont été de même tendues. L'administration Bush a certes essayé d'inverser cette tendance, mais le succès a été très limité. Le pouvoir politique est parvenu dans un premier temps à écarter les militaires des décisions de recourir à la force contre l'Irak en 2003 aux États-Unis. Les grandes délibérations dans le «cabinet de guerre» du 21 décembre 2002 ou celles qui eurent lieu le 25 janvier 2003 semblent avoir été menées sans que les représentants des forces armées aient véritablement pu imposer leurs positions, leurs craintes et leurs doutes ${ }^{100}$. Le responsable du Central Command (Centcom), à qui incombait en cas de guerre la responsabilité de la conduite des opérations, a émis plusieurs réserves à l'égard du déclenchement des hostilités, mais il n'était pas isolé ; il a été suivi par les chefs des quatre armées de la Navy, des marines, de l'Air Force et de l'Army101, ainsi que le Secrétaire d'État Colin Powell, l'ancien chef d'État-major conjoint des armées. Commandant en chef des opérations armées durant la guerre du Golfe, le général SCHWARZKOPF a souligné, à propos du Secrétaire à la Défense Donald RuMSFELD : « Certains de ses propos m’ont inquiété. [...] Dans ses commentaires, il paraît qu'il ne tient pas compte de l'armée ». Alors que Donald RUMSFELD a entendu imposer depuis 2001 une dynamique visant à " transformer » l'appareil de défense en concentrant les militaires sur leurs missions traditionnelles et en les éloignant du nation-building, sa volonté de restaurer pleinement l'autorité civile sur les militaires s'est retrouvée confrontée à l'enlisement en Irak et, par la suite, sa propre démission.

Un autre exemple marquant des relations, parfois tumultueuses, entre le Chef de l'État et l'armée est, dans tous les cas, celui de la France qui, durant toute la période du XIXe siècle et de la première partie du $\mathrm{XX}^{\mathrm{e}}$ siècle, mettait le plus en avant les principes de subordination et d'apolitisme. Pour ce qui est de la subordination, d'une part, " [1]a force armée », écrit le juriste DuGUIT, « doit être un instrument passif entre les mains du gouvernement. Celui-ci ne peut remplir sa mission que s'il dispose de la force armée; or disposer de la force armée, c'est pouvoir s'en servir comme d'une force matérielle inconsciente. Cela exclut la possibilité pour les commandants de la force armée de refuser, sous un prétexte quelconque, d'obtempérer aux ordres du gouvernement... Il n'y aurait plus d'État si les chefs militaires pouvaient discuter les ordres qu'ils reçoivent du gouvernement. L'idéal serait que la force armée fut une machine inconsciente que le gouvernement pourrait mettre en mouvement en pressant un bouton électrique »102. Pour ce qui concerne, d'autre part, le second aspect, certaines pratiques, comme la privation du droit d'électorat et

100 E. LAURENT, La Guerre des Bush, Paris, Pocket, 2003, p. 139 et s.

101 T. LindemAnN, « Des guerriers pour faire la paix. L'armée américaine en Irak », Cultures \& Conflits, 2007, pp. 20-21.

102 Cité par J. DE SoTo, « Pouvoir civil et pouvoir militaire», in: La défense nationale Paris, Presses Universitaires de France, 1958, p. XX; voy. R. GIRARDET. «Pouvoir civil et pouvoir militaire dans la France contemporaine », Revue française de science politique, 1960, p. 5. 
d'éligibilité imposée aux militaires sous la III République entendaient garantir l'apolitisme des armées. Cependant, plus fondamentalement, l'apolitisme revient à spécifier que l'armée doit exécuter sans discuter ${ }^{103}$. Cela étant, en France, en échange de la non-immixtion dans les affaires politiques par l'armée, de facto, le gouvernement ne s'ingérait pas dans la conduite des affaires militaires, sauf par le biais de l'allocation des crédits militaires, d'où la grande influence sur les politiques étrangère et militaire ${ }^{104}$. En mars 1936, lors de la remilitarisation de la Rhénanie, le point de vue du Général GAMELIN, chef d'État-major général, plutôt réticent à une action militaire, s'impose sans difficulté devant celui du Président du Conseil, Albert SARRAUlT, et du ministre des affaires étrangères, PierreÉtienne FLANDIN, favorables quant à eux à une intervention. Autrement dit, le pouvoir politique ne fixait toujours pas clairement les grandes lignes d'une guerre, alors que lui seul détenait une connaissance interne de la situation politique que le chef militaire, simple spécialiste, ne pouvait percevoir ${ }^{105}$, et ce, jusqu'à la fin de la IVe République.

La $\mathrm{V}^{\mathrm{e}}$ république constitue une rupture décisive, un tournant majeur dans les relations entre le pouvoir politique et les chefs militaires. L'article 15 de la Constitution pose juridiquement le principe de la subordination du pouvoir militaire au pouvoir civil. À ce titre, l'article 5.2 du règlement de la discipline générale dans les armées fixe que le " commandement de certaines formations procède des pouvoirs du Président de la République et est exercé en son nom ». Le Chef de l'État assume la responsabilité grâce à son État-major des grandes orientations sans nécessairement solliciter l'avis des chefs militaires, devenus des auxiliaires soumis à l'autorité politique. L'État major particulier est dirigé par un officier général, soit le chef d'état-major particulier, avec lequel le Président s'entretient quotidiennement, et est composé d'au moins six officiers supérieurs des différentes armées qui composent les forces militaires françaises. Comme le synthétise à nouveau Samy CoHEN «les généraux de la Ve République n’ont pas la chance qu'avaient leurs ainés » ${ }^{106}$. Cependant, l'état de supériorité du président a été généralement accepté et " un modus videndi s'est dégagé qui se reflète dans la bonne entente évidente lors de la guerre du Golfe, mais aussi lors des interventions en Afrique ${ }^{107}$. L'Opex résulte aujourd'hui d'une décision politique du pouvoir exécutif, déclinée, au niveau militaire concret au travers d'une chaine de commandement confirmée par l'ordonnance $\mathrm{n}^{\circ}$ 59-147 du 7 janvier 1959 «portant organisation générale de la défense » qui transforme la volonté politique en un ordre du chef d'état-major des armées (CEMA) ou, le cas échéant, du directeur général de la gendarmerie nationale, dans un cadre national, multinational ou sous mandat international.

103 J. MEYNAUD, op. cit., p. 77.

104 S. COHEN, « Le pouvoir politique et l'armée », Pouvoirs, 2/2008, p. 20.

105 Idem ; voy. J. MEYNAUD, op. cit, p. 77.

106 S. COHEN, op. cit., p. 20. Voy. plus généralement S. COHEN, La défaite des généraux. Le pouvoir politique et l'armée sous la Ve République, Paris, Fayard, 1994, 276 p. mais aussi V. PORTERET, État-nation et professionnalisation des armées: les députés français face au déclin de l'armée de masse de 1962 à nos jours, Paris, L'Harmattan, 2005, 342 p.

107 S. COHEN, « Le pouvoir politique et l'armée », Pouvoirs, 2/2008, p. 28. 


\section{Conclusion}

On peut aisément remarquer que peu importe la structure gouvernementale, le Chef de l'État a la charge symbolique des armées, et c'est, dans tous les cas, au pouvoir exécutif de mobiliser les troupes. Dans le système présidentiel américain, les relations entre l'exécutif et le législatif sont par nature profondément différentes des équilibres institutionnels qui caractérisent les régimes parlementaires. Pourtant, il faut d'emblée remarquer la prédominance de l'exécutif en matière de défense dans tous les États et la forme d'immunité juridictionnelle, au niveau interne, dont jouit la décision d'intervenir militairement. $\mathrm{Au}$ niveau international, toutefois, on aura tôt fait d'identifier les bornes juridiques qui existent depuis la fin de la seconde guerre mondiale au travers le droit coutumier et la Charte des Nations Unies qui prohibent l'emploi de la force.

Certes, le degré d'autonomie de l'Exécutif varie. On remarque aussi que, dans les anciennes puissances coloniales, les gouvernements sont généralement autorisés à déployer des troupes sans l'accord du parlement. C'est le cas de la France, du Royaume-Uni, de l'Espagne, de la Belgique et de la Grèce ${ }^{108}$. Au contraire, le consentement du Parlement s'impose en Allemagne, en principe, en Autriche, au Danemark, Suède, Irlande et dans les différents États d'Europe de l'est. D'ailleurs, en Hongrie, il faut les deux tiers des membres du Parlement pour autoriser le déploiement de troupes. En Italie et aux Pays-Bas, ce n'est que par tradition politique, sans obligation constitutionnelle, que l'approbation du parlement est requise.

Certains États, comme l'Allemagne et le Japon, ont pris en compte la dimension internationale des opérations militaires en interprétant leurs textes constitutionnels d'une manière plus souple. La prise en compte de cette dynamique mondiale se retrouve dans d'autres pays. Ainsi, en Suède, si l'opération prend place dans une mission mise sur pied par une organisation internationale, le consentement parlementaire n'est plus requis. L'on constate dès lors une influence réelle de la société internationale sur l'organisation interne des pouvoirs militaires.

Plus fondamentalement, la nature substantiellement différente des conflits, la conjugaison du développement technologique, nucléaire et aérospatial et les réformes engagées depuis le milieu des années $90^{109}$, après la chute du mur de Berlin, ouvrent la voie à un nouveau modèle d'armée, réduit en effectifs et beaucoup moins éloigné qu'auparavant des

108 W. WAGNER, « The democratic control of military power Europe », Journal of European Public Policy, 2006, p. 204.

109 Voy. F. CAilleteau, « Les structures à l'épreuve des faits : leçons de la guerre du Golfe », R.F.A.P., 1992, p. 221-231. 
préoccupations et des contraintes s'imposant à l'ensemble des administrations d'État, mettant en quelque sorte fin à « l'exception militaire » 110 .

Nous étions partis, dans notre introduction, de l'image de ce prince aux côtés de ces hommes armés des temps jadis. La défense nationale était assumée par le Roi ; sinon son statut et son maintien n'étaient pas garantis. Il devait donc être le chef guerrier, $d u x$ bellorum, pour être le chef politique. Ceci a disparu dans les sociétés contemporaines puisque désormais il faut être chef politique pour être chef militaire. Cette inversion du postulat, partant de la désignation constitutionnelle du Chef de l'État comme le chef des armées, suit la doctrine de CLEMENCEAU, pour qui « [1] guerre [...] est une chose trop grave pour la confier à des militaires ».

110 J.-F. BUREAU. « La réforme militaire en France : une mutation identitaire », Politique étrangère, 1997, pp. 6981. 